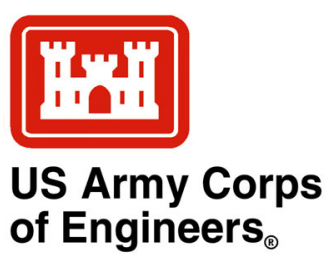

\title{
Video-Based Wave Direction Measurements in a Scale Physical Model
}

\author{
by William R. Curtis, Kent K. Hathaway, \\ K. Todd Holland, and William C. Seabergh
}

PURPOSE: The Coastal and Hydraulics Engineering Technical Note (CHETN) herein describes a method for video-based measurement of wave direction in an idealized coastal inlet physical model. This method may be applied in other physical model settings or extended to field measurement applications. Seabergh, Curtis, and Thomas (2002) give a detailed description of the method, validation statistics, and application to a variety of physical model experiments.

BACKGROUND: At the U.S. Army Engineer Research and Development Center's (ERDC's) Coastal and Hydraulics Laboratory (CHL), a 1:50 undistorted scale idealized coastal inlet physical model was constructed under the Coastal Inlets Research Program (CIRP). CIRP conducts research to reduce operations and maintenance costs at inlet navigation projects. Among the many applications of the facility, the model is used to investigate the complex interaction of incident wind-waves with variations in inlet bathymetry, engineered inlet structures, and tidal currents (Smith et al. 1998; Seabergh 1999). In the physical model setting, the primary diagnostic tools to measure hydrodynamic processes such as scaled wind-waves and currents are capacitive wave rods and acoustic doppler velocimeter (ADV) probes. These instruments are deployed at discrete locations to measure wave height and current fields. The drawback to the application of wave rods and ADV probes is that they are intrusive and may cause localized disturbance in the fluid motions of the model. Also, relocation of gauges can be time consuming and requires additional experimental runs. To minimize these undesired perturbations, CIRP applied a video-based nonintrusive technique to accurately measure wave parameters with high spatial and temporal resolution. This technique provides measurements of vector-mean wave direction of time and space varying scale wind-wave fields observed on the model's free surface. These measurements have broad application and can provide quantitative information for understanding first-order coastal inlet process, as well as support computational model development and validation.

VIDEO TECHNIQUE: During the past decade, video techniques have been applied in the field to measure variations in coastal hydrodynamic processes (Holland, Holman, and Sallenger 1991; Lippmann and Holman 1991; Holland et al. 1995; Holland and Holman 1996; Hathaway and Bottin 1997; Holland and Holman 1999; Stockdon and Holman 2000). These techniques, as well as the present study, build on photogrammetric and image processing techniques developed by the Argus Research Program coordinated by the Coastal Imaging Lab, Oregon State University (Holman et al. 1993; Aarninkhof and Holman 1999). The physical model's video system consists of obliquely looking monoscopic camera stations mounted at vantage points above the physical model. Lens distortion, camera locations and ground control coordinates are measured to establish a geometric model that relates observation in two-dimensional (2-D) image 
coordinates $(u, v)$ to the physical model's three-dimensional (3-D) xyz-coordinate system via a direct linear transformation. Figure 1 shows the geometric relationship for an observation in image space and object space, where $\left(x_{c}, y_{c}, z_{c}\right)=$ camera position, $(u, v)=$ observation on the image plane, $(\tau, \sigma, \phi)=$ camera rotation angles and $f=$ lens focal length. The ability to accurately transform between image- and object-space enables the cameras to operate as a metric system. For a comprehensive treatise on the mathematics of the camera calibration and application of photogrammetric methods, the reader is referred to Holland et al. (1997).

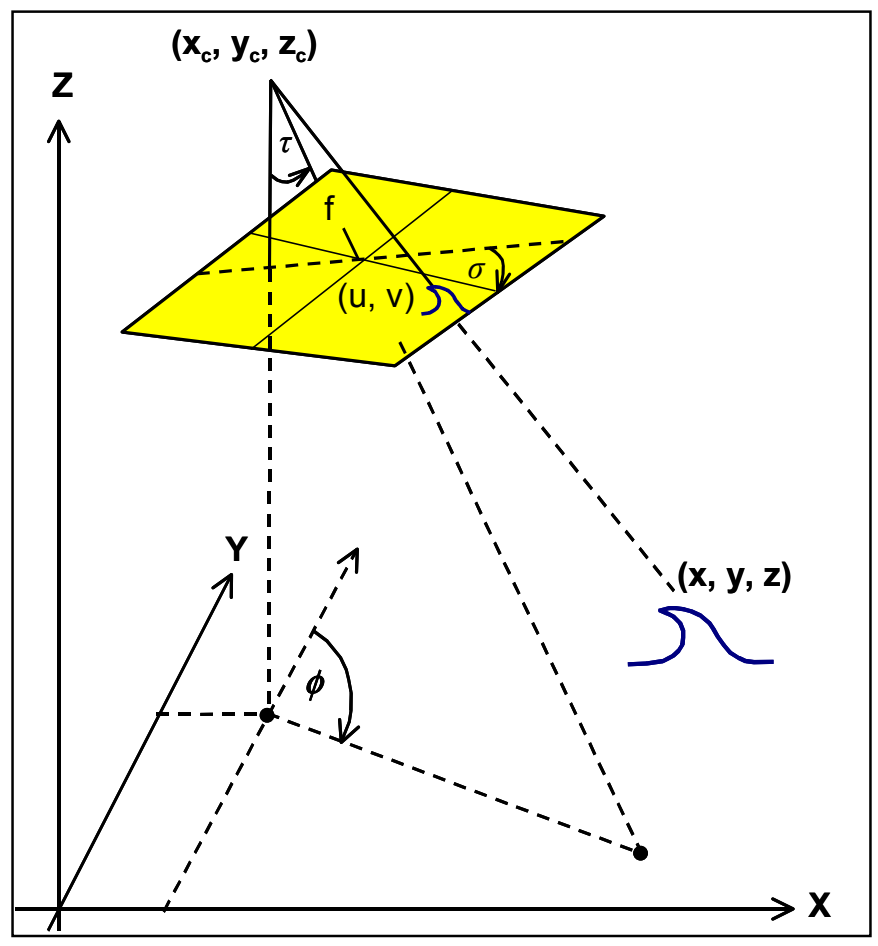

Figure 1. Geometric relationship between image and object space

The general premise for video-based wave direction measurement in the physical model is as follows. Diffuse lamps create a directional light gradient over the model. As waves propagate by a fixed point on the free surface, the intensity of directional specular reflection from the surface of the wave slope varies from light to dark (Figure 2). The degree of variation depends on the viewing angle relative to the direction of wave propagation and illumination. For example, a vertical camera angle would observe a field of high and low intensity bands over the free surface as waves propagate toward the illumination source. That is, the forward face of the wave would have a higher intensity signature (illuminated) than the back face (shadowed). Temporal and spatial variations in light intensity are observed by a camera and recorded on videotape. Signals are digitized and analyzed to determine the relationship between the phase structure (time lag) of light intensity time series records measured at specified locations within the camera's field of view (Figure 3). It is assumed that the physical relationship between the visual signature of the wave in image space and the true wave in object space is spatially constant. 


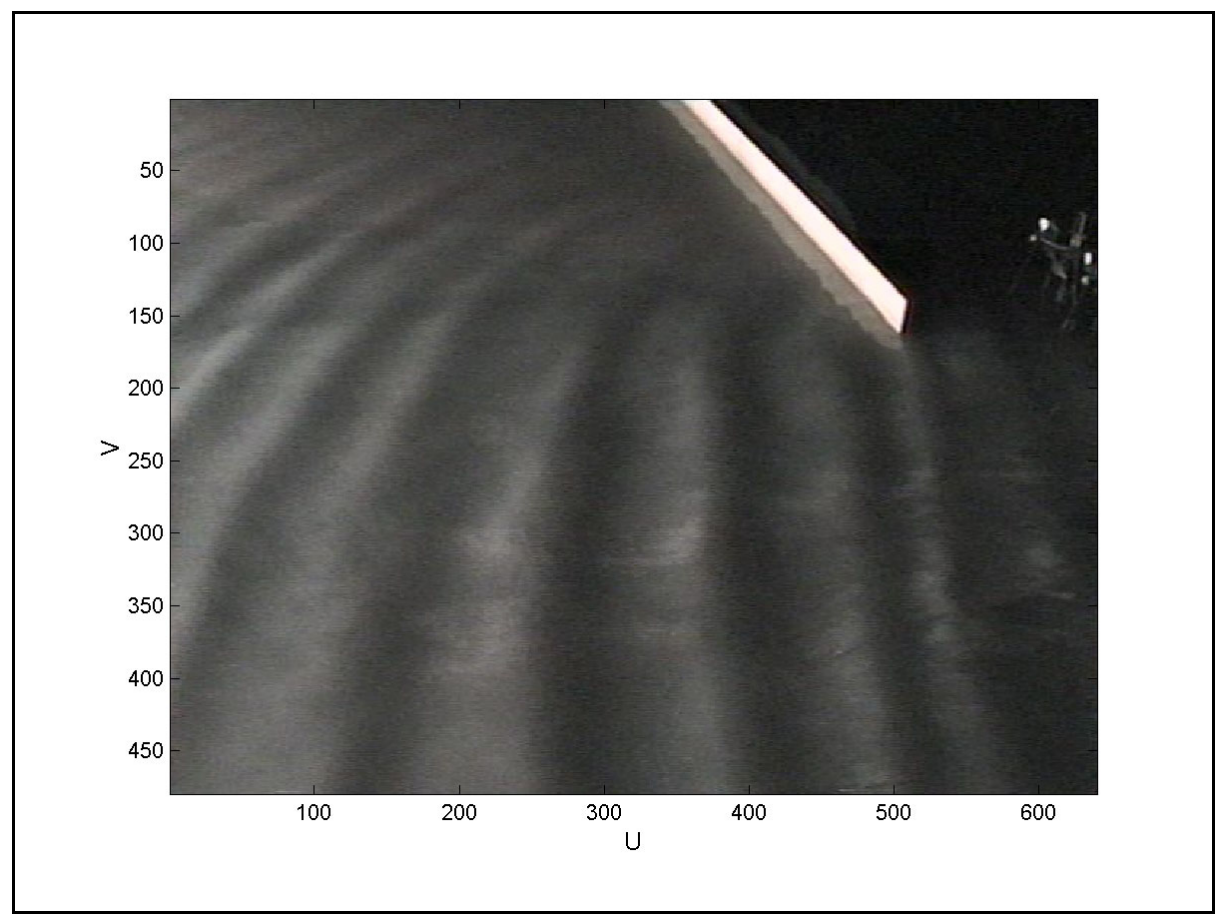

Figure 2. Video snapshot during random wave experiment. High intensity bands are the illuminated forward-facing surface wave slopes

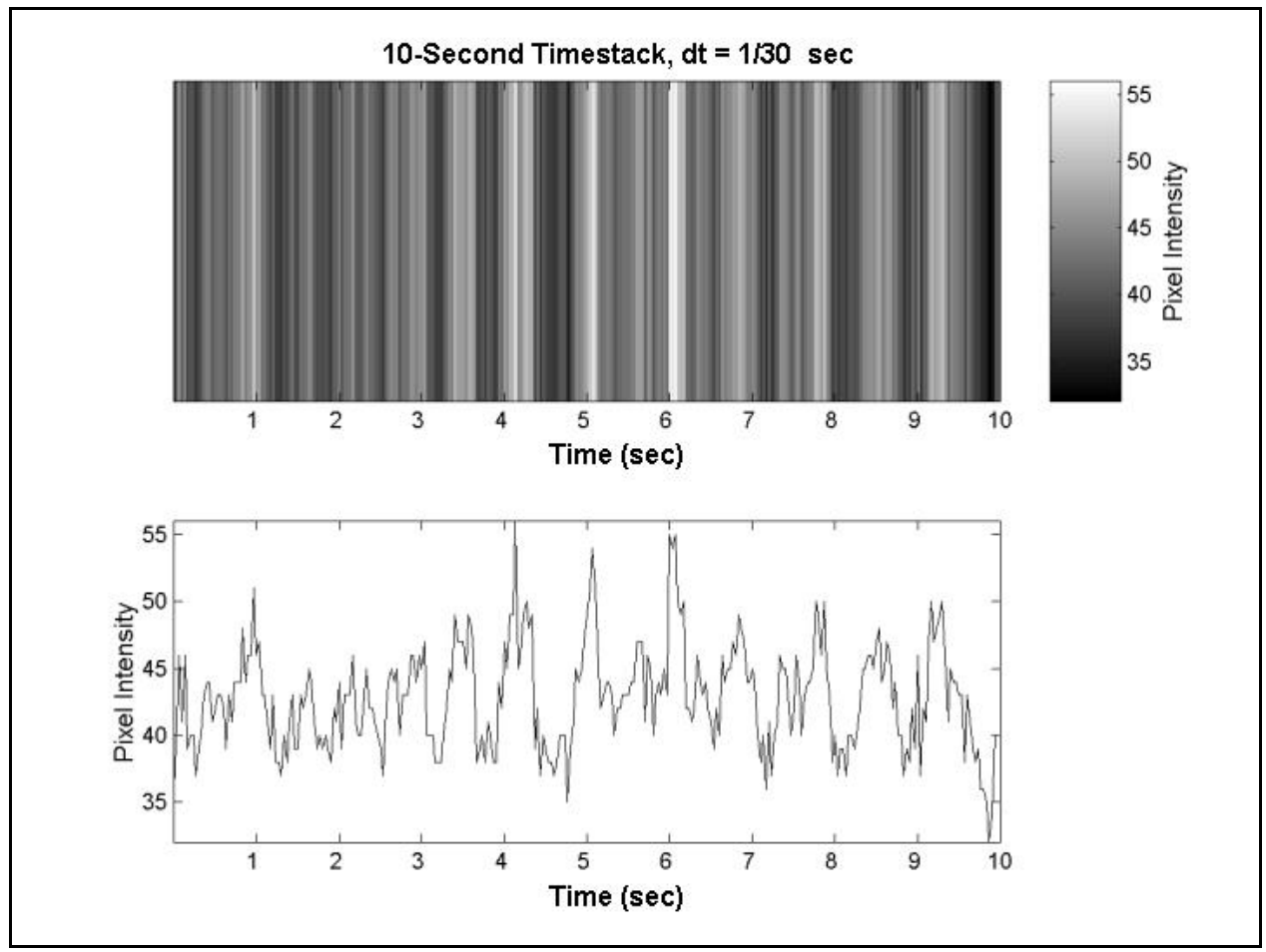

Figure 3. Example 10-sec duration timestack (pixel intensity time series record) 
Data are acquired by commercially available video components. For the experimental arrangement discussed herein, two synchronized Sony ${ }^{\mathrm{TM}}$ SSC-54A color video CCD cameras are mounted at vantage points above the physical model on support beams. Synchronization of the cameras is required for simultaneous observations from each camera view. Manual iris lenses with a focal length of $8 \mathrm{~mm}$ are used due to the range of the cameras from the target area and the desired degree of spatial resolution. A typical camera orientation results in $<2 \mathrm{~cm}$ pixel resolution in the direction perpendicular to the camera azimuth over the entire imaged area. Pixel footprints in the direction of the camera azimuth are highly resolved in both the near and far fields $(<4 \mathrm{~cm})$.

Camera-lens combinations are calibrated at the ERDC Field Research Facility to determine lens distortion coefficients and optical characteristics of a given camera-lens combination prior to installation. Each camera is connected to a SVHS videocassette recorder (VCR) by composite video cable. SVHS cable is desired for increased videotape resolution, however long cable lengths between cameras and VCRs $(>30 \mathrm{~m})$ in the laboratory dictates the use of composite video cable to prevent loss of signal strength. The video signal from each camera passes through a synchronized vertical interval time code (VITC) generator before being recorded by the VCR at a frame rate of $30 \mathrm{~Hz}$. VITC time code is burned onto each video frame to allow identification of a specific frame of interest during VCR playback and image digitization.

Following equipment installation, horizontal and vertical coordinates of the camera locations and ground control points (GCPs) are surveyed. GCPs are landmarks observed in a camera view that have known object space and image space coordinates. A geometry solution can be determined for each camera view with as few as two GCPs. However, more GCPs allow for a least-squares solution with minimal error. A geometry solution was calculated for each camera orientation for access by the image processor and coordinate transformation algorithms.

Diffuse incandescent lamps are positioned to illuminate the model's free surface create a clearly visible signature of the wave field by the camera. Care is taken to prepare the area around and above the physical model to minimize undesired shadows and light reflections in the field of view. During beta testing, lamplight refracted through the clear water column onto the model floor. As waves propagated across the measurement area, refracted high intensity bands of light dominated the video signal and degraded the phase structure of the desired surface wave signal. To prevent light refraction, hollow glass spheres were mixed throughout the water column making the water opaque. The spheres have the appearance of a white powder and a range of specific gravity of $0.1-1.5 \mathrm{~g} / \mathrm{cc}$.

A variety of laboratory random wave and monochromatic wave conditions have been measured with video near the influence of an idealized breakwater, jetty, and inlet channel/bay system. For the purpose of this CHETN, three wave experiments are evaluated to measure wave diffraction patterns near an idealized breakwater. These experiments include two random wave cases $\left(f_{\text {peak }}=25 \mathrm{~Hz}\right.$ and $\left.0.56 \mathrm{~Hz}\right)$, and a single monochromatic case $\left(f_{\text {peak }}=1.25 \mathrm{~Hz}\right)$ (Figure 4). Wave fields are generated by a unidirectional wave paddle rotated 20 deg relative to the breakwater axis. For each experiment, an idealized field of wave gage arrays; each comprised of 11 pixel sampling elements was designed to provide estimates of mean wave direction at high spatial resolution within the physical model. Based upon the generated target spectra and mean water 
depth, a $0.8 \mathrm{~m} \times 0.8 \mathrm{~m}$ aperture cross-shaped array was designed to optimize measurement of expected wavelengths. The wave phenomena of interest occur in a 3-D physical model coordinate system. However, the waves are observed with a 2-D video image. Knowing only the camera geometry established using GCPs, 2-D image coordinates cannot be directly transformed to physical model coordinates. To solve the problem of coordinate transformation, information in addition to the camera geometry solution must be known. Assuming that sampling elements are located on the free surface of the physical model, still-water level (SWL) and the camera geometry solution are combined to make the direct linear transformation between the 2-D and 3-D coordinate systems. Thus, each array element has a unique $x y$-coordinate and a common $z$-coordinate equivalent to SWL in the physical model's reference frame.

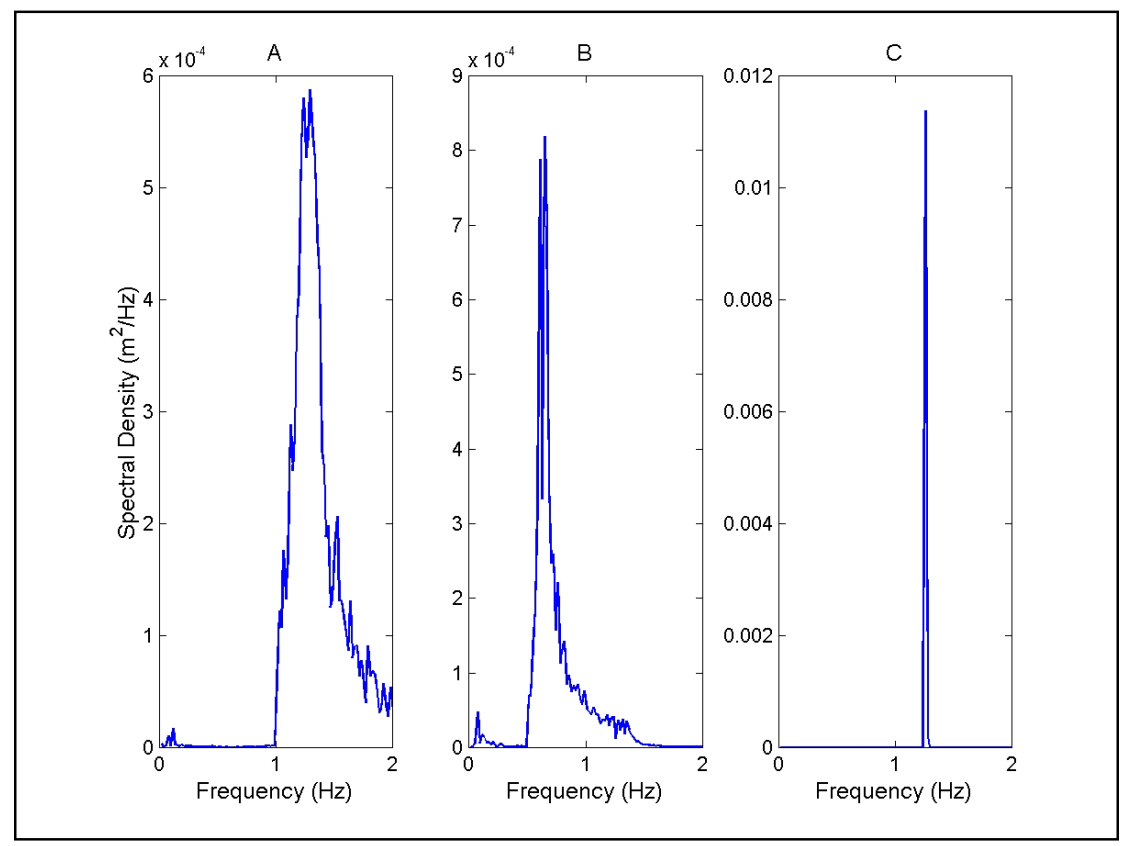

Figure 4. Target spectra measured at generator by capacitive wave rod

Pixel arrays are located to cover the region of interest in each camera view. For the idealized breakwater laboratory arrangement, two camera views are used to image the area of interest. One camera (ID = Camera 4) has 1,133 pixel sampling elements (103 measurement arrays), and the other (ID = Camera 6) has 814 sampling elements (74 measurement arrays). Camera views overlap slightly, and combination of the two views results in 82 unique video-based directional measurement locations. Figure 5 shows the superposition of the elements $(+)$ on a rectified image of a wave field obtained from Camera 4 during a spectral wave experiment. Circles indicate pixel array locations that are collocated with in situ sensors (ADV probe and capacitive wave rod).

Recorded video of the wave experiment is played back to the image processor for digitization. The platform for the image processing software is a Silicon Graphics Inc ${ }^{\mathrm{TM}} \mathrm{R} 5000$ video work station. The highest frequency that can be defined by the video sampling rate of $30 \mathrm{~Hz}$ is $15 \mathrm{~Hz}$. Because the high-frequency limits of the target spectra were sufficiently lower than the band limiting frequency $(<70$ percent), no effort was made to remove information contained in the original analog signals. During the playback process, the image processor collects time series 
records of pixel intensity at locations corresponding to measurement array elements. Pixel time series records are commonly known as timestacks. The software reads VITC recorded onto the each video frame to locate the image records of interest.

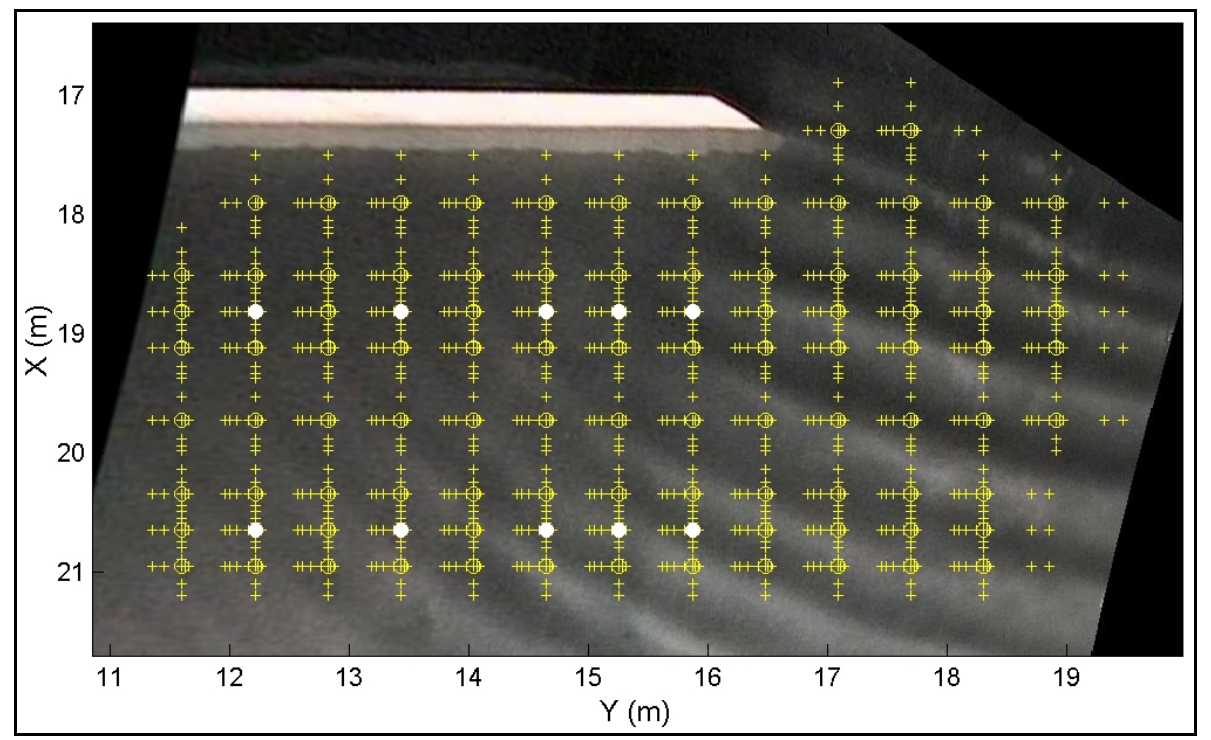

Figure 5. Pixel array element locations for Camera 4

TIME SERIES ANALYSIS: The premise for use of video as a wave direction metric system is that for waves propagating past a fixed point on the free surface, the intensity of directional specular reflection from the wave slope varies from light to dark. It is assumed that outside the wave-breaking region, the phase structure of the timestacks is a proxy for the phase structure of the true propagating wave signal. Timestacks are analyzed to provide estimates of vector-mean wave direction, $\theta_{m}(f)$. Directions are derived from the phase difference between the signals measured at one location relative to the signal at another for all array elements. With the separation distance between pixel array elements known in real-world coordinates, timestack records are analyzed in terms of an estimated root-mean-square average wave number, $k_{r m s}(f)$ following Herbers, Elgar, and Guza (1995). This method is based on an expansion of the theoretical cross-spectrum of gravity waves for small sensor separations relative to wavelength. The computed wave number moments are extended to calculations of alongshore and cross-shore components of wave number, $k_{y}(f)$ and $k_{x}(f)$ by linear combination of normalized quadspectra:

$$
k_{x}=\sum_{p=1}^{N} \sum_{q=1}^{N} \alpha_{p q} \frac{Q_{p q(f)}}{\sqrt{P_{p p}(f) P_{q q}(f)}}
$$

where $Q_{p q}=$ quadspectrum of pixel pair at $\left(x_{p}, y_{p}\right)$ and $\left(x_{q}, y_{q}\right)$ and $N=$ number of array elements. Least squares fit solutions of wave number coefficients $\left(\alpha_{p q}\right)$ are obtained by singular value decomposition of

$$
\sum_{p=1}^{N} \sum_{q=1}^{N} \alpha_{p q} i^{n} \frac{\left(x_{p}-x_{q}\right)^{n-m}\left(y_{p}-y_{q}\right)^{m}}{(n-m) ! m !}=1
$$


for $n=2, m=0$ and $n=2, m=2$, and

$$
\sum_{p=1}^{N} \sum_{q=1}^{N} \alpha_{p q} i^{n} \frac{\left(x_{p}-x_{q}\right)^{n-m}\left(y_{p}-y_{q}\right)^{m}}{(n-m) ! m !}=0
$$

for $n$ and $m=$ other. Note, $i=\sqrt{-1}$.

Normalized co- and quadspectra are estimated by Fourier transform of sensor pair timestacks. Each timestack is $7 \mathrm{~min}$ in duration and consists of 12,600 samples. To improve computational performance, frequencies above $3 \mathrm{~Hz}$ are not considered for analysis. Timestacks are linearly detrended, tapered and divided into six ensembles of 2,048 samples and band averaged resulting in a frequency resolution of $0.0439 \mathrm{~Hz}$ and $36 \mathrm{deg}$ of freedom.

Noisy pixel intensity signals and low coherence between sampling elements of pixel arrays was anticipated as arising from two primary sources. First, generated wave spectra result in a broad range of wave heights in the diffraction region behind the breakwater, with some waves lacking a visual signal. Second, a gradient in diffuse light intensity illuminated the measurement area. Therefore, low amplitude and low variance pixel intensity fluctuations may be observed across a poorly illuminated array. High pixel intensity and low variance may be observed across array elements in brightly lit areas. The coherence-squared function was calculated for all possible gage pairings in an array. A coherence-squared threshold amplitude value of 0.7 is arbitrarily selected. Only frequency content of array pairings meeting or exceeding the threshold is accepted to calculate estimates of $\theta_{\mathrm{m}}(f)$ with reliability. Generally, $\theta_{\mathrm{m}}(f)$ is estimated with confidence at the spectral peak.

For validation, collocated ADV observations of velocities are sampled at a rate of $20 \mathrm{~Hz}$ for duration of $204.8 \mathrm{sec}$, resulting in 4,096 samples. ADV time series records were linearly detrended, tapered, and ensemble averaged resulting in a frequency resolution of $0.078 \mathrm{~Hz}$ and $32 \mathrm{deg}$ of freedom with a maximum frequency cutoff of $5 \mathrm{~Hz}$.

RESULTS: Video measurements of $\theta_{\mathrm{m}}(f)$ are validated against collocated in situ ADV probe measurements for the three wave experiments. To facilitate comparison, ADV energy density and direction values are interpolated using a piecewise cubic Hermite interpolation within the frequency bands observed by the video system. Figure 6 presents a representative graphical comparison of normalized energy density and direction versus frequency at a single measurement location for a random wave experiment $\left(f_{\text {peak }}=1.25 \mathrm{~Hz}\right)$.

Several statistics are selected to intercompare mean wave directions obtained near the peak of the spectra for the two measurement techniques. Statistical results for the idealized breakwater experiment include mean angular difference $(<\Delta \theta>)=-2.65 \mathrm{deg}$, standard deviation of mean angular difference $\left(\sigma_{\theta}\right)=4.46 \mathrm{deg}$, root-mean-square error $\left(\mathrm{RMSE}_{\theta}\right)=4.46 \mathrm{deg}$, scatter index $\left(\mathrm{SI}_{\theta}\right)=8$, and correlation coefficient $\left(\mathrm{R}_{\theta}\right)=0.92$. Figure 7 shows a scatter plot of wave directions at the spectral peak for collocated video and ADV observations. The statistics demonstrate excellent correlation with low scatter indices. Mean angular difference values may be considered scalar wave direction bias. Although biased low, the wave directions are within 
the reported margin of error for the ADV probe measurements, which are accurate to within 5 deg.

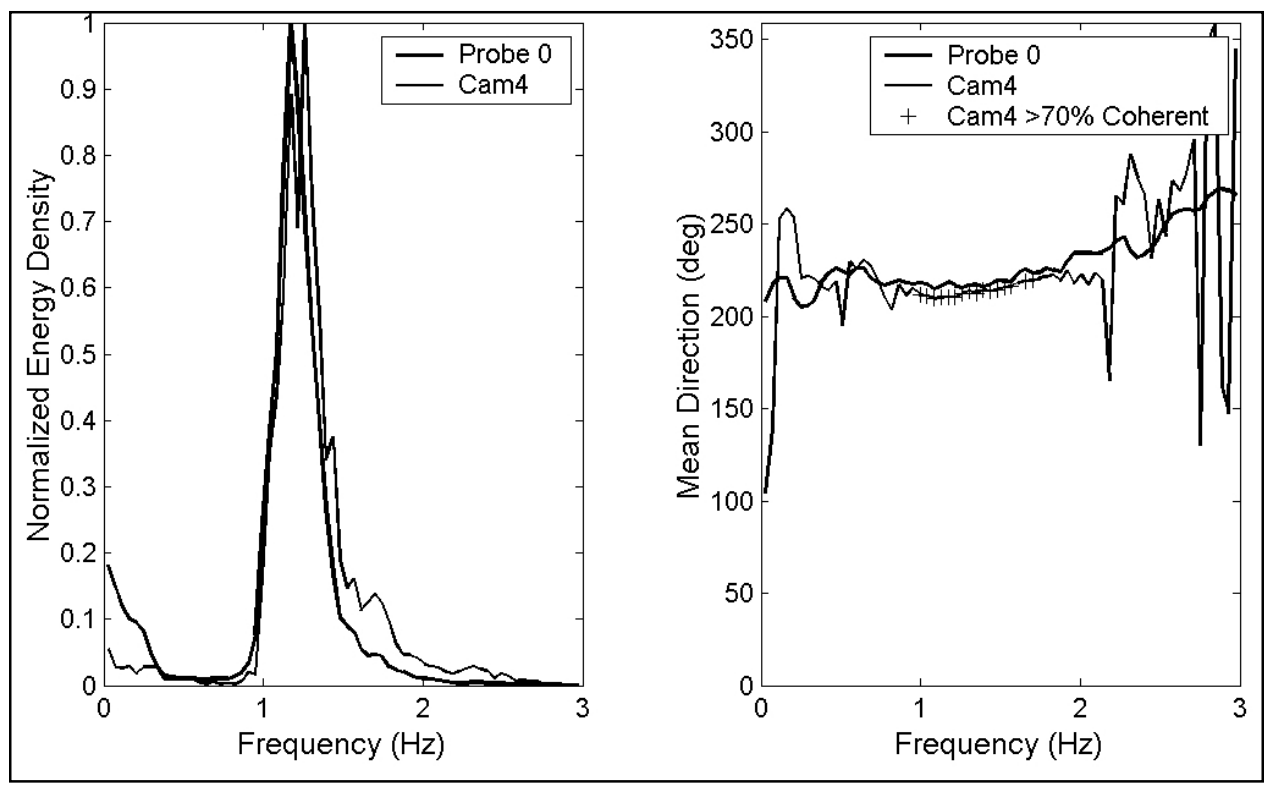

Figure 6. Representative collocated video-ADV spectra

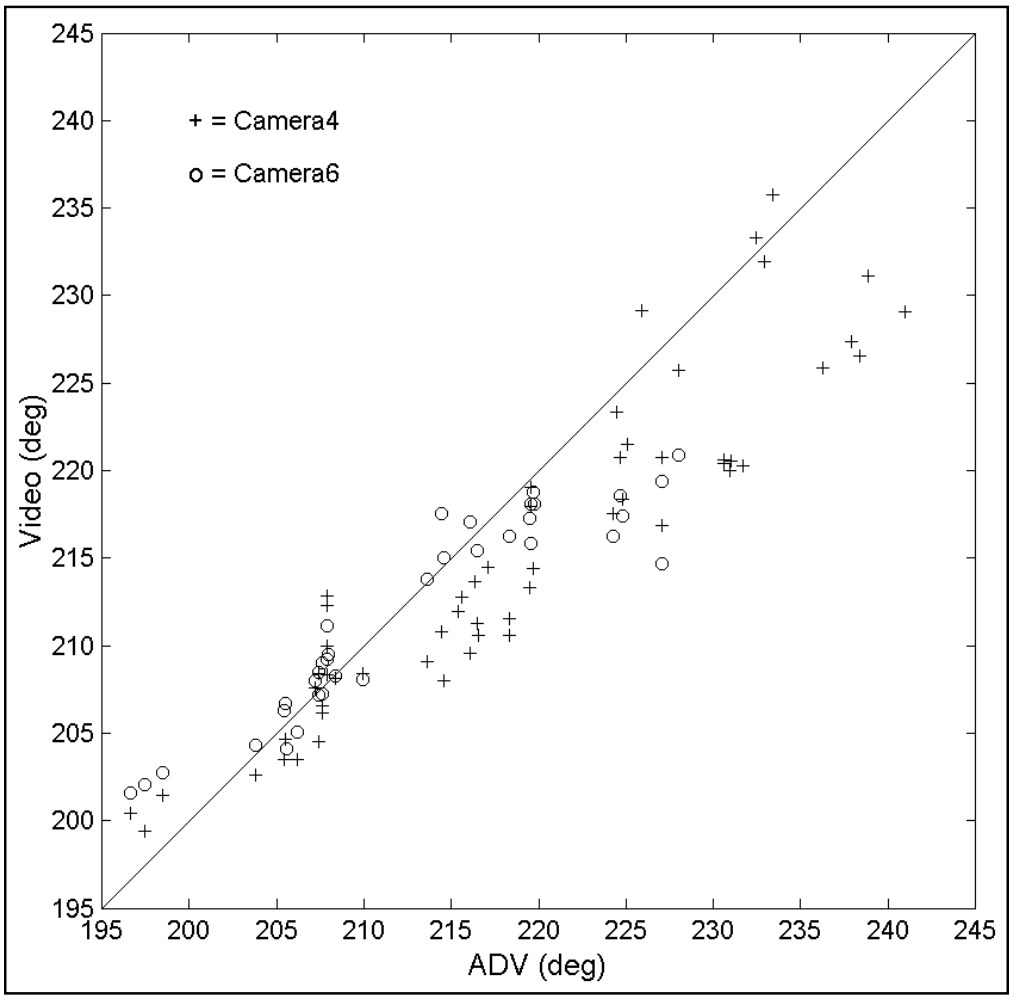

Figure 7. Video-ADV intercomparison of peak vector-mean wave direction 
To demonstrate the video system's application, peak mean wave direction for the random wave experiment $f_{\text {peak }}=1.25 \mathrm{~Hz}$ is indicated by arrows (scaled by wave celerity) superimposed on a rectified snapshot of the wave field in Figure 8. Arrows with a circled tail represent directions obtained from analyses meeting the strict coherence cutoff criterion. Some arrows without circled tails are spurious in direction and magnitude in the diffraction area behind the breakwater. Where wave height is significantly reduced or in regions of the measurement area that are poorly illuminated, the visual signal of the wave degrades due to lack of contrast. Image contrast may be improved by adjustment of the lens iris, improved directional illumination, and use of a monochromatic camera system.

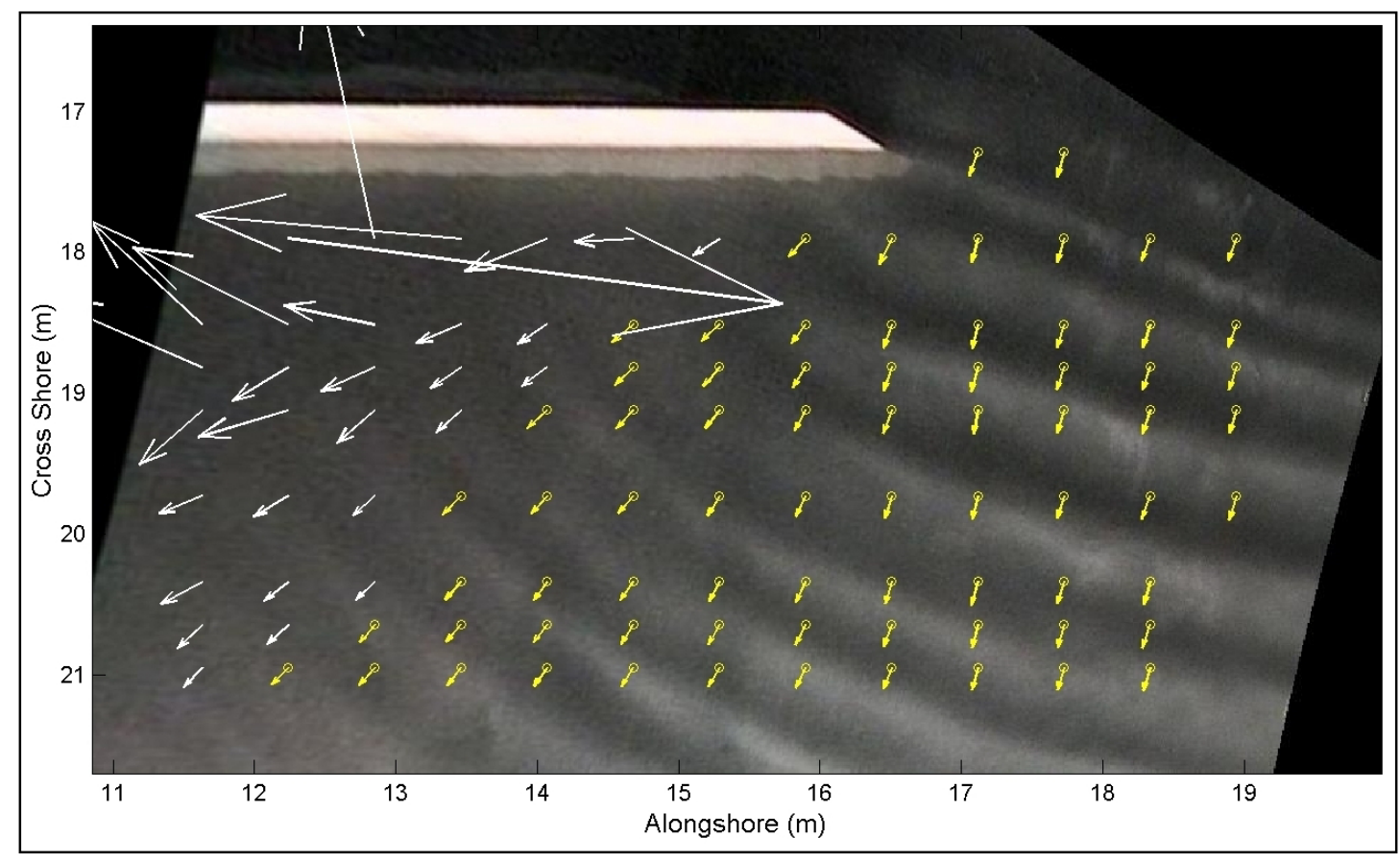

Figure 8. Peak vector-mean wave direction, $f_{\text {peak }}=1.25 \mathrm{~Hz}$

CONCLUSION: Spatially dense measurement of wave direction in the physical model setting is a labor and equipment-intensive activity that necessitates relocation of instrumentation and regeneration of the wave time series. Additionally, in situ or contacting methods of wave measurement cause localized perturbations in the wave field that may not be desired. Thus, CIRP was motivated to develop and implement a video-based wave measurement method in an idealized coastal inlet physical model.

The wave measurement system was developed with commercially available hardware and standard image processing and photogrammetric techniques to provide spatially dense measures of wave direction over a large area of interest. Laboratory application and validation of this system represents an advance in wave measurement technology. Experimentation confirms that the system provides spatially dense and accurate measures of wave direction over a large area of interest, which is not economically obtained by standard in situ methods. 
ADDITIONAL INFORMATION: Questions about this CHETN can be addressed to Mr. William R. Curtis (601-634-3040; e-mail: William.R.Curtis@erdc.usace.army.mil) or to Mr. Kent K. Hathaway (252-261-6840; e-mail: Kent.K.Hathaway@erdc.usace.army.mil). For information about the Coastal Inlets Research Program, please contact the Program Manager, Dr. Nicholas C. Kraus (601-634-2016; e-mail: Nicholas.C.Kraus@erdc.usace.army.mil). This Technical Note should be cited as follows:

Curtis, W.R., Hathaway, K.K., Holland, K.T., and Seabergh, W.C. (2002). "Videobased wave direction measurements in a scale physical model," Coastal and Hydraulics Engineering Technical Note ERDC/CHL CHETN IV-49, U.S. Army Engineer Research and Development Center, Vicksburg, MS.

http://chl.wes.army.mil/library/publications/chetn

\section{REFERENCES}

Aarninkhof, S., and Holman, R. A. (1999). "Monitoring the nearshore with video," Backscatter 5-11, May 1999.

Hathaway, K. K., and Bottin, R. R. (1997). "Video measurement of wave runup on coastal structures," CETN-VI-28, U.S. Army Engineer Waterways Experiment Station, Vicksburg, MS.

Herbers, T. H. C., Elgar, S., and Guza, R. T. (1995). Generation and propagation of infragravity waves, Journal of Geophysical Research 100, 24,863-24,872.

Holland, K. T., Holman, R. A., and Sallenger, A. H. (1991). "Estimation of overwash bore velocities using video techniques." Proceedings, Coastal Sediments '91. American Society of Civil Engineers, 489-497.

Holland, K. T., Raubenheimer, B., Guza, R. T., and Holman, R. A. (1995). "Runup kinematics on a natural beach," Journal of Geophysical Research 100(C3), 4985-4993.

Holland, K. T., and Holman, R. A. (1996). "Field observations of beach cusps and swash motions," Marine Geology 134, 77-93.

Holland, K. T., Holman, R. A., Lippman, T. C., Stanley, J., and Plant, N. (1997). "Practical use of video imagery in nearshore oceanographic field studies," IEEE Journal of Ocean Engineering 22(1), 81-92.

Holland, K. T., and Holman, R. A. (1999). "Wavenumber-frequency structure of infragravity swash motions," Journal of Geophysical Research 106(C6), 13,479-13,488.

Holman, R. A., Sallenger, A. H., Lippmann, T. C., and Haines, J. W. (1993). "The application of video image processing to the study of nearshore processes," Oceanography 6(3), 78-85.

Lippmann, T. C., and Holman, R. A. (1991). "Phase speed and angle of breaking waves measured with video techniques." Proceedings, Coastal Sediments '91. ASCE, 543-556.

Seabergh, W. C. (1999). "Physical model for coastal inlet entrance studies," Coastal and Hydraulics Engineering Technical Note, CHETN IV-19, U.S. Army Engineer Research and Development Center, Vicksburg, MS.

Seabergh, W. C., Curtis, W. R., and Thomas, L. J. (2002). "Physical model study of wave diffractionrefraction at an idealized inlet," Technical Report ERDC/CHL TR-02-27, U.S. Army Engineer Research and Development Center, Vicksburg, MS.

Smith, J. M., Seabergh, W. C., Harkins, G. S., and Briggs, M. J. (1998). "Wave breaking on a current at an idealized inlet," Technical Report CHL-98-31, U.S. Army Engineer Waterways Experiment Station, Vicksburg, MS.

Stockdon, H., and Holman, R. A. (2000). "Estimation of wave phase speed and nearshore bathymetry from video imagery," Journal of Geophysical Research 105(C9), 22,015-22033. 